\title{
Effects of Functionalization and Stress on Graphene Electronic Properties: Focusing on Bandgap
}

\author{
Xiaohan Ming* \\ School of Physics, Huazhong University of Science and Technology, Wuhan, China
}

\begin{abstract}
Graphene is considered as a promising base material for nanodevices due to the excellent mechanical, thermal and electronic properties. However, when developing 2D semiconductor device such as a field-effect transistor, one obstacle we are facing is the zero bandgap of pure graphene, which makes it hard to apply to the semiconductor field. In this study, we verify the feasibility of opening the bandgap by functionalizing a graphene and adding stresses based on first principle calculations, where hydroxyl and epoxy groups are used. The effect on bandgap is also observed in the calculations after adding stress about a few GPa. The results show that bandgaps of $1 \sim 2 \mathrm{eV}$ in functionalized graphene were opened and stresses of $1 \mathrm{GPa}$ induced slightly variations of bandgaps. The electron density differences indicate that the loaded functional groups take away the charge of graphene, making it a betatopic system. Our study may provide a potential method to modify the electronic properties of two-dimensional materials.
\end{abstract}

\section{Introduction}

The development of artificial intelligence (AI) and the fifth-generation mobile communication technologies spawns the demand of next-generation electron devices which requires miniaturization, low energy consumption and high frequency [1-2]. A traditional Si-based complementary metal-oxide-semiconductor (CMOS) transistor has a theoretical limit of $5 \mathrm{~nm}$ due to the rapidly decrease of carrier mobility with the thickness [3-6]. In recent years, some two-dimension materials, including transition metal disulfide (TMD) compounds and their heterojunction based field-effect transistors (FETs), have been proposed [7-9]. For instance, Desai et al. [4] prepared double-layer MoS2 FET, and found that a gate length of $\sim 1 \mathrm{~nm}$ can still realize switch, indicating a good prospect as channel device.

Graphene also has the potential to become the ideal replacement for the silicon-based nanodevice because of the superior electronic and mechanic properties [10-11]. For example, its charge carriers act like massless Dirac fermions which can switch between electrons and holes under certain voltage, and its special structure makes it unnecessary to rearrange its atoms for adjusting to external stress. Due to its unique properties, thousands of scientist dive into the possible application of graphene. However, unlike TMDs as direct-gap semiconductors, the zero bandgap of graphene prohibits many promising applications in the semiconductor field. It will be helpful for us to develop a regulative bandgap which can be modulated by the external influence that we exert.

Many researchers explored the methods for opening the bandgap of graphene. Specifically, Ke et al. [12] used $30 \mathrm{GPa}$ pressure on trilayer graphene and a $2.5 \mathrm{eV}$ bandgap is acquired. Monteverde et al. [13] also observed bandgap opening in rippled graphene by stressing. Nemnes et al. [14] calculated the band structure of a graphene putting on a $\mathrm{HfO} 2$ substrate. They found that zero bandgap of the Hf exposed systems but a $2 \mathrm{eV}$ bandgap of the $\mathrm{O}$ exposed ones. Da et al. [15] experimentally showed that graphene bandgap can be opened by changing the stacking pattern.

In this study, we demonstrate the effects of functional groups and stress to modulate bandgap of graphene based on a first principle calculation. CASTAP module in MS is adopted to calculate the band structures of graphene when adding epoxy and hydroxyl groups under a given pressure. The calculation results show that the groups opened the bandgap of graphene by about $1.5 \mathrm{eV}$, and the stress can also slightly affect the bandgap. To explain the underlying mechanism of functional groups, we calculated the charge transfer between graphene and the groups.

\section{Methods}

All the calculations and geometry optimizations were performed in the CASTAP module of Material Studio 7.0, which is based on the first-principles pseudopotential plane-wave methods and the density functional theory (DFT). We set the cutoff energy to $10 \mathrm{eV}$ and the quality to medium for all calculation. More specifically, we choose the generalized gradient approximation (GGA) and the Perdew- Burke- Ernzerhof (PBE) function for the geometry optimization before adding any stress to the structure and choose the local density approximation (LDA) with CA-PZ function for the structure relaxations after adding stress and for all 
band structure calculations. In order to avoid unreasonable structural changes, we limit the stress under $1 \mathrm{GPa}$.

\section{Results and discussions}

The calculated models of pure graphene $(G)$, graphene with epoxy groups (GO) and graphene with hydroxyl groups $(\mathrm{GOH})$ are shown in Figure 1 (a-c). A $2 \times 2$ supercell of graphene is used and only one functional group is loaded on a graphene. If defining the number ratio of the functional group and the bravais lattice as the concentration of the functional group, the concentrations in the present models are 0 for $\mathrm{G}$ and $25 \%$ for $\mathrm{GO}$ and $\mathrm{GOH}$, which is a moderate concentration as suggested in Ref. [16]. Meanwhile, the stability of epoxy and hydroxyl groups on graphene was also confirmed [16]. As shown, the $\mathrm{O}$ atom in $\mathrm{GO}$ is bonded with two adjacent $\mathrm{C}$ atoms. However, $\mathrm{O}$ atom in $\mathrm{GOH}$ connects with only one $\mathrm{C}$ because of the coordinate hydrogen atom. The bonding difference of two groups indicates the discrepancies of binding energy and charge transfer. Then, to explore the effect of stress, tensile stress and compressive stress were applied on $\mathrm{G}, \mathrm{GO}$ and $\mathrm{GOH}$ along $\mathrm{x}$ and $\mathrm{y}$ directions.

Table 1 shows the lattice parameters of $\mathrm{G}, \mathrm{GOH}$ and GO under compressive stress and tensile stress. To avoid significant and unreasonable transformations of graphene, only the stress less than $1 \mathrm{GPa}$ is used. For the case of $0 \mathrm{GPa}$, the lattice parameter is $2.454 \AA$, which is very close with the standard value of $2.46 \AA$ [17]. Then, under the compressive stresses of 0.5 and $1 \mathrm{GPa}$, the parameters decrease to $2.449 \AA$ and $2.446 \AA$. After exerting tensile stress of 0.5 and $1 \mathrm{GPa}$, the parameters increase to $2.467 \AA$ and $2.476 \AA$. Slightly deformation like this is inevitable when a graphene contacts with a surface or under a temperature gradient [18-19]. When there is no stress on GO, the lattice parameter is $2.463 \AA$ which is slightly larger than G. This might be because of the double bonded $\mathrm{O}$ atom, which enlarges the distance between two adjacent $\mathrm{C}$ atoms. However, the lattice parameter of $\mathrm{GOH}$ of zero stress becomes smaller owing to the upwarping of bonding $\mathrm{C}$ atom, as shown in Fig. 1. The effects of the external stress for $\mathrm{GO}$ and $\mathrm{GOH}$ are similar with $\mathrm{G}$, where the compressive stress decreases and tensile stress increases the lattice parameters.
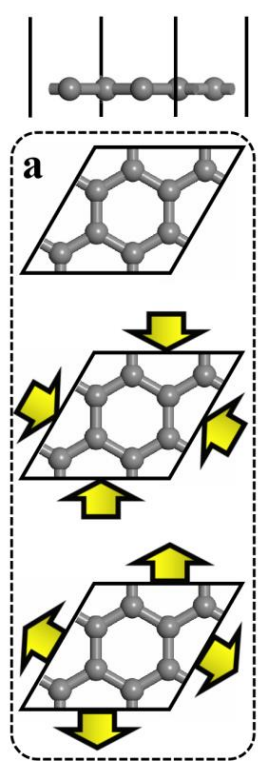
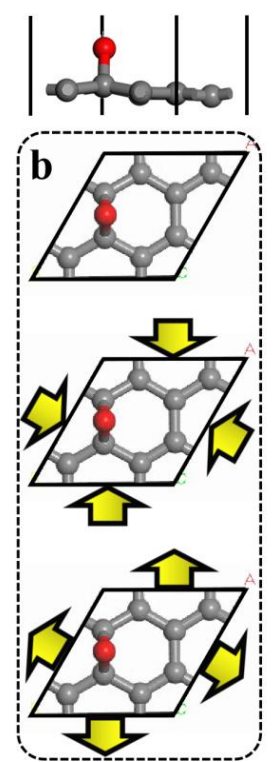

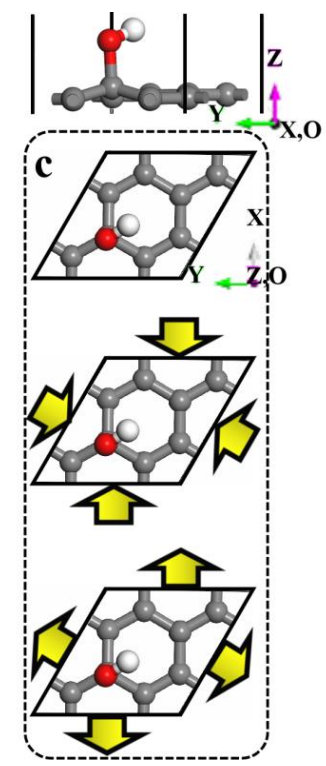

Fig. 1. Structures of graphene modified by functional groups and stress, where (a) pristine graphene (G), (b) graphene with epoxy groups $(\mathrm{GO})$ and $(\mathrm{c})$ graphene with hydroxyl groups $(\mathrm{GOH})$ under well relaxation, tensile stress and compressive stress are displayed.

Table 1. Lattice constant variations of G, GOH and GO with the external stresses, where positive and negative stresses indicate compressive stress and tensile stress on the systems.

\begin{tabular}{cccc}
\hline \multirow{2}{*}{ Stress $(\mathrm{GPa})$} & \multicolumn{3}{c}{ Lattice parameter $(\AA)$} \\
\cline { 2 - 4 } & $\mathrm{G}$ & $\mathrm{GO}$ & $\mathrm{GOH}$ \\
\hline 1 & 2.446 & 2.443 & 2.427 \\
0.5 & 2.449 & 2.451 & 2.436 \\
0 & 2.454 & 2.462 & 2.447 \\
-0.5 & 2.467 & 2.475 & 2.461 \\
-1 & 2.476 & 2.486 & 2.471 \\
\hline
\end{tabular}


Band structures and density of states (DOSs) for G, $\mathrm{GO}$ and $\mathrm{GOH}$ under well relaxation are shown in Fig. 2 (a-c). The highly symmetric paths and points used in band plots are also displayed in Fig. 2 (d). For the case of G, Dirac cone in the path of HK is found, representing the zero bandgap of pure graphene [20]. What's more, the zero DOS at Fermi energy also declares this property. However, clear gaps between conduction and valence bands for GO and GOH are shown when epoxy group and hydroxyl group were applied. For example, a bandgap of $\sim 1.5 \mathrm{eV}$ of GO is found in Fig. 2 (b). The DOS around Fermi energy is also close to zero, presenting a semiconductor property. Similarly, $\sim 0.9 \mathrm{eV}$ bandgap for $\mathrm{GOH}$ system is also acquired. The significant effects of groups on bandgap of graphene indicate that functionalization is a feasible method to modify the graphene band structure.

To find the underlying mechanism of functional groups on graphenes, we calculated the charge distribution of $\mathrm{G}, \mathrm{GO}$ and $\mathrm{GOH}$. Figure 3 is a visual representation of the electron density differences. The purple part indicates the charge accumulation while the yellow part indicates the charge depletion. Figure 3 (a) demonstrates the stable and periodic structure of sixmembered ring of graphene. Electrons accumulate in the $\mathrm{C}-\mathrm{C}$ bands but deplete in the circle center. However, in Fig. 3 (b), the epoxy group destroys the charge balance in the annulus. The strong electronegativity of $\mathrm{O}$ atom tends to carry off the electrons of $\mathrm{C}$ atoms. Clearly, the bonding electrons between $\mathrm{O}$ atom and $\mathrm{C}$ atoms are closer to $\mathrm{O}$ and make the graphene betatopic. Then, the charge transfer decreases the electroconductivity of graphene and opens the band gap. Similar result is also found in Fig. 3 (c) still because of the strong electronegativity of $\mathrm{O}$ atom. The difference is that bonded with an $\mathrm{H}$ atom, $\mathrm{O}$ atom in hydroxy group can only bond one $\mathrm{C}$ atom, and thus the charge transfer becomes weak. It may be the reason why the bandgap of $\mathrm{GO}$ is larger than $\mathrm{GOH}$.

As mentioned above, when applying a twodimensional material, such as graphene, the complex surrounding may exert stresses on it. Understanding the band structure of a graphene under a given stress is important for the practical applications. Figure 4 shows the bandgap of $\mathrm{G}, \mathrm{GO}$ and $\mathrm{GOH}$ varying with stress. When there are no functional groups added to the graphene ( $\mathrm{G}$ case), the stress cannot open the bandgap, indicating a slight effect on band structure. After attaching the functional groups, the opened bandgap becomes fluctuant with no certain trend. To be specific, both the compressive and tensile stresses lead to larger bandgaps of GO than zero stress, but all of them are around $1.5 \mathrm{eV}$. For $\mathrm{GOH}$, the stresses mainly decrease the bandgap except $-0.5 \mathrm{GPa}$, and the variation is negligible. It could be obviously acknowledged that the external stress does change the bandgap while the effect of stress is not systematic and has no strong regularity. With concerns about excessive and unreasonable deformations of the graphene system, we do not apply stress over $1 \mathrm{GPa}$ to the system which leads to the irregular effect of external stress.
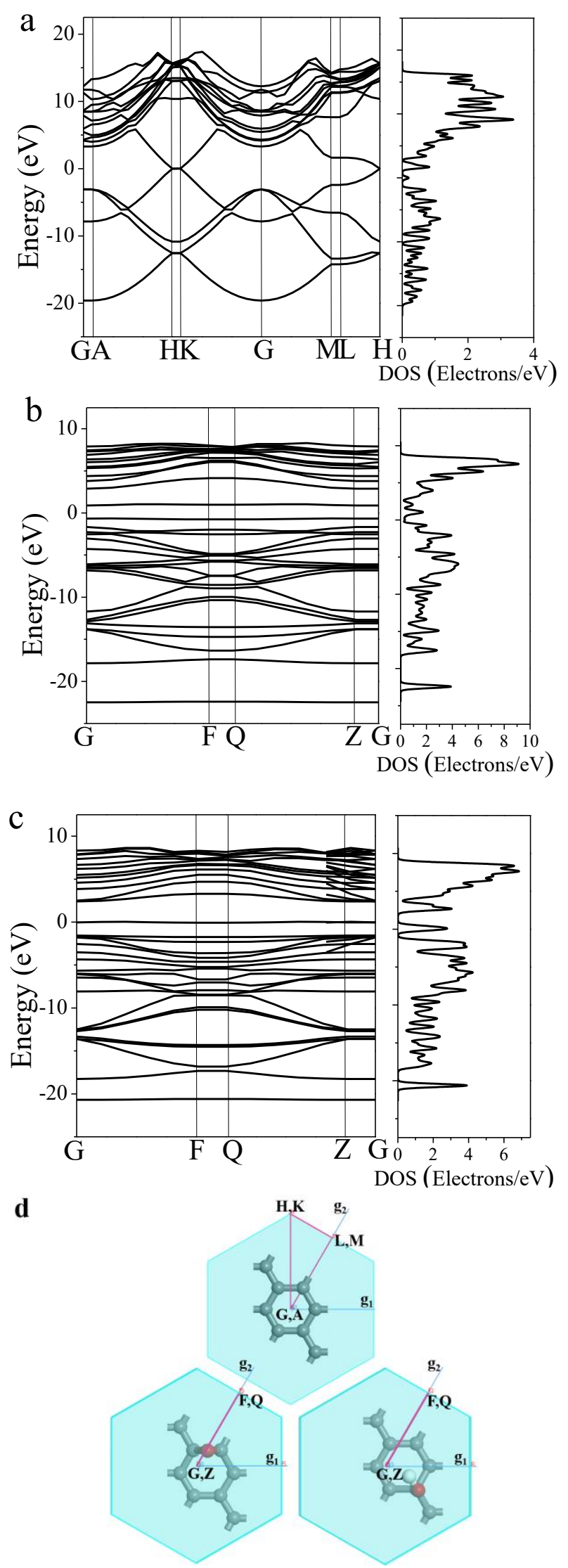

Fig. 2. Band structures and density of states (DOSs) of (a) G, (b) GO and (c) GOH under well relaxation, and (d) is the highly symmetric paths and points in First Brillouin zone. 


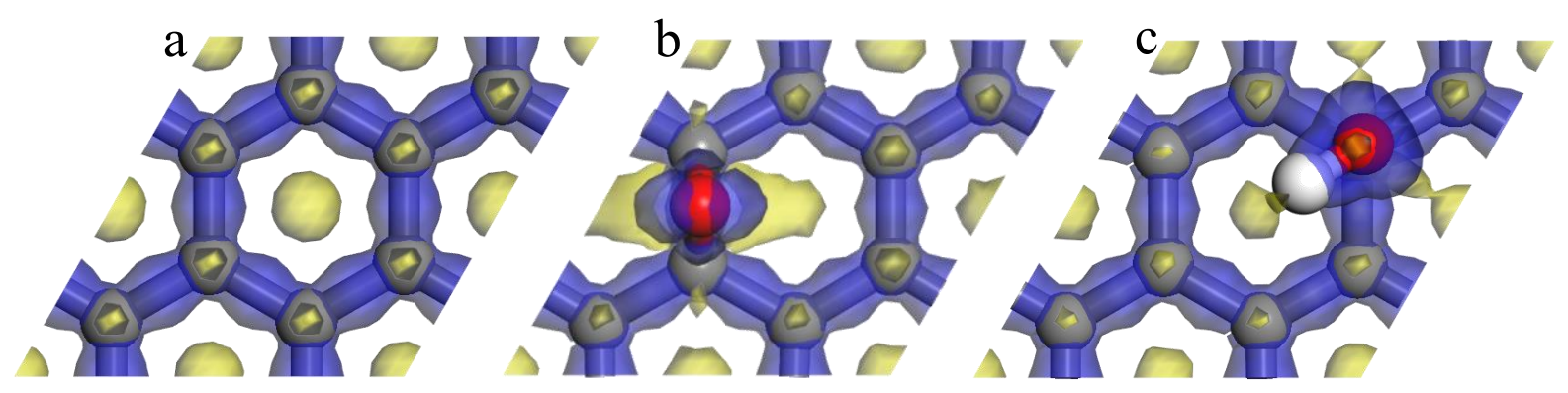

Fig. 3. Electron density difference plots for GO and GOH under well relaxation.Band structures and density of states (DOSs) of (a) G, (b) GO and (c) GOH under well relaxation, and (d) is the highly symmetric paths and points in First Brillouin zone.

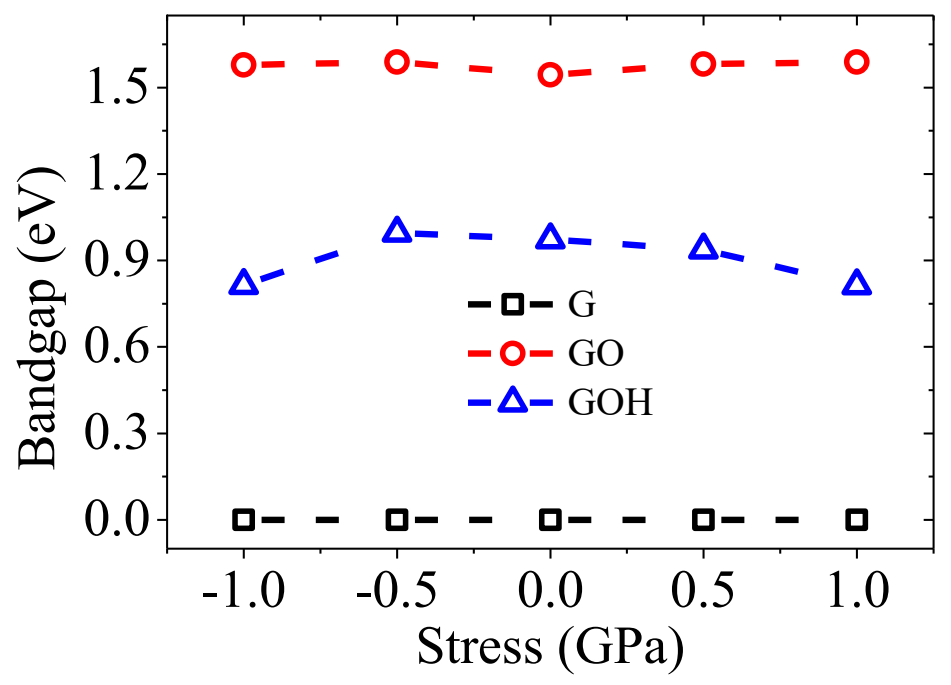

Fig. 4. Bandgap of G, GOH and GO changing with external stresses.

\section{Conclusion}

In this study, we adopted hydroxyl and epoxy groups to open the bandgap of graphene and the effect of external stress was also discussed based on first principles calculation. By using a $2 \times 2$ graphene supercell and loading only one functional group, we got functionalized graphene with reasonable group concentrations. Then, structure optimizations were performed for G, GO and $\mathrm{GOH}$, and the lattice parameters of $\mathrm{GO}$ and $\mathrm{GOH}$ are slightly higher and lower than $G$, respectively. The compressive and tensile stresses can lead to minor deformations of all systems. The band structure results show that the pure graphene is zero bandgap semiconductor while the hydroxyl and epoxy groups can open the gap of graphene by $\sim 0.9$ and $1.5 \mathrm{eV}$. The charge transfers due to the difference of functional groups were also obtained. We found that the strong electronegativity of $\mathrm{O}$ atom makes graphene become betatopic systems for both hydroxyl and epoxy groups. Stresses less than $1 \mathrm{GPa}$ were applied on G, GO and $\mathrm{GOH}$ to investigate the bandgap variations. Constant zero and slight fluctuations of bandgap for pure and functionalized graphene indicate a weak effect of stress on band structure.

\section{References}

1. Deblina Sarkar, Wei Liu, Xuejun Xie, et al. MoS2 Field-Effect Transistor for Next-Generation LabelFree Biosensors. Acs Nano, 2014, 8(4):3992.

2. Godfrey Akpakwu, Bruno Silva, Gerhard P. Hancke, et al. A Survey on $5 \mathrm{G}$ Networks for the Internet of Things: Communication Technologies and Challenges. IEEE Access, 2017, 5(12):3619-3647.

3. Kawaura H, Sakamoto T, Baba T . Observation of source-to-drain direct tunneling current in $8 \mathrm{~nm}$ gate electrically variable shallow junction metal-oxidesemiconductor field-effect transistors. Applied Physics Letters, 2000, 76(25):3810.

4. Desai S B , Madhvapathy S R, Sachid A B , et al. MoS2 transistors with 1-nanometer gate lengths. ence, 2016, 354(6308):99-102.

5. Liu L, Lu Y, Guo J . On Monolayer, Field-Effect Transistors at the Scaling Limit. IEEE Transactions on Electron Devices, 2013, 60(12):4133-4139.

6. Yoon Y, Ganapathi K, Salahuddin S . How good can monolayer MoS2, transistors be?. Nano Letters, 2011, 11(9):3768-3773. 
7. Feng Zhang. On the Tunability of Short-Channel Effects in MoS2 Field-Effect Devices. Nano Letters, 2014, 15(1):301.

8. Bilu Liu, Yuqiang Ma, Anyi Zhang, et al. High Performance WSe2 Field-Effect Transistors via Controlled Formation of In-Plane Heterojunctions. Acs Nano, 2016, 10(5):5153.

9. Fathipour S, Hwang W S, Kosel T, et al. Exfoliated MoTe2 field-effect transistor. Device Research Conference. IEEE, 2013.

10. Park S J , Kwon O S, Lee S H, et al. Ultrasensitive Flexible Graphene Based Field-Effect Transistor (FET)-Type Bioelectronic Nose. Nano Letters, 2012, 12(10):5082-5090.

11. Kim S, Nah J, Jo I, et al. Realization Of A High Mobility Dual-gated Graphene Field-effect Transistor With Al_2o_3 Dielectric. Applied Physicsletters, 2009, 94(6):161-163.

12. Ki F, Chen Y, Yin K, et al. Large bandgap of pressurized trilayer graphene, 2019, 116(19): 91869190.

13. Monteverde U, et al. (2015) Under pressure: Control of strain, phonons and bandgap opening in rippled graphene. Carbon 91:266-274.

14. Nemnes G A, Dragoman D, Dragoman M . Graphene bandgap induced by ferroelectric Pca21
HfO2 substrate: a first-principles study. Physical Chemistry Chemical Physics, 2019.

15. Da Zhan, Jia Xu Yan, Zhen Hua Ni, et al. BandgapOpened Bilayer Graphene Approached by Asymmetrical Intercalation of Trilayer Graphene. Small, 2015, 11(9-10):1177-1182.

16. Musso T, Kumar P V , Foster A S, et al. Graphene Oxide as a Promising Hole Injection Layer for MoS2-Based Electronic Devices. Acs nano, 2014, 8(11):11432-9.

17. Matthew J Allen, Vincent C Tung, Richard B Kaner. Honeycomb Carbon: A Review of Graphene. Chemical Reviews, 2009, 110(1):132-145.

18. H. Sevinçli, Topsakal M , Durgun E, et al. Electronic and magnetic properties of $3 \mathrm{~d}$ transitionmetal atom adsorbed graphene and graphene nanoribbons $[\mathrm{J}]$. Physical Review B, 2008, 77(19):3107-3109.

19. Kundalwal S I , Meguid S A , Weng G J . Strain gradient polarization in graphene[J]. Carbon, 2017, 117(Complete):462-472.

20. Morales-Torres, Sergio, Pastrana-Martínez, Luisa, Figueiredo, José. Design of graphene-based TiO photocatalysts-a review. Environmental Science \& Pollution Research, 2012, 19(9):3676-3687. 